\title{
BMJ Open Health-related quality of life in congenital heart disease surgery patients in Pakistan: protocol for a mixed- methods study
}

\author{
Laila Akbar Ladak, ${ }^{1}$ Babar Sultan Hasan, ${ }^{2}$ Janice Gullick, ${ }^{3}$ Khadija Awais, ${ }^{4}$ \\ Ahmed Abdullah, ${ }^{4}$ Robyn Gallagher ${ }^{1,5}$
}

To cite: Ladak LA, Hasan BS, Gullick J, et al. Health-related quality of life in congenital heart disease surgery patients in Pakistan: protocol for a mixedmethods study. BMJ Open 2017;7:e018046. doi:10.1136/ bmjopen-2017-018046

- Prepublication history for this paper is available online. To view these files, please visit the journal online (http://dx.doi org/10.1136/bmjopen-2017018046).

Received 2 June 2017

Revised 10 August 2017

Accepted 25 September 2017

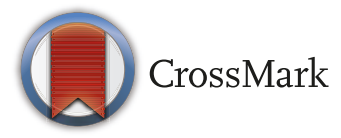

${ }^{1}$ Charles Perkins Centre, Faculty of Nursing, The University of Sydney, Camperdown, New South Wales, Australia ${ }^{2}$ Department of Pediatrics and Child health, The Aga Khan University, Karachi, Sindh, Pakistan

${ }^{3}$ Faculty of Nursing, The University of Sydney,

Camperdown, New South Wales, Australia

${ }^{4}$ Medical College, The Aga

Khan University, Karachi, Sindh, Pakistan

${ }^{5}$ Faculty of Health, University of Technology, Sydney, New South Wales, Australia

Correspondence to

Laila Akbar Ladak;

llad8915@uni.sydney.edu.au

\section{ABSTRACT}

Introduction Reduced health-related quality of life (HRQOL) has been reported in postoperative patients with congenital heart disease (CHD). However, there is a paucity of data from low-income and middle-income countries (LMIC). Differences in sociodemographics and sociocultural contexts may influence HRQOL. This protocol paper describes a study exploring HRQOL in surgical patients with CHD from a tertiary hospital in Pakistan. The study findings will assist development of strategies to improve $\mathrm{HRQOL}$ in a resource-constrained context.

Methods and analysis This prospective, concurrent triangulation, mixed-methods study aims to compare HRQOL of postsurgery patients with CHD with age-matched healthy siblings and to identify HRQOL predictors. A qualitative component aims to further understand $\mathrm{HRQOL}$ data by exploring the experiences related to $\mathrm{CHD}$ surgery for patients and parents. Participants include patients with CHD (a minimum of $\mathrm{n} \sim 95$ ) with at least 1-year postsurgery follow-up and no chromosomal abnormality, their parents and age-matched, healthy siblings. PedsQL 4.0 Generic Core Scales, PedsQL Cognitive Functioning Scale and PedsQL 3.0 Cardiac Module will measure HRQOL. Clinical/surgical data will be retrieved from patients' medical files. Student's t-test will be used to compare the difference in the means of HRQOL between $\mathrm{CHD}$ and siblings. Multiple regression will identify HRQOL predictors. A subsample of enrolled patients $(n \sim 20)$ and parents $(n \sim 20)$ from the quantitative arm will be engaged in semistructured qualitative interviews, which will be analysed using directed content analysis. Anticipated challenges include patient recruitment due to irregular follow-up compliance. Translation of data collection tools to the Urdu language and back-translation of interviews increases the study complexity.

Ethics and dissemination Ethics approval has been obtained from The Aga Khan University, Pakistan (3737-Ped-ERC-15). Study findings will be published in peer-reviewed journals and presented at national and international conferences.

\section{INTRODUCTION}

Childhood is a critical period in growth and development, forming the basis for healthy adult life. Therefore, when congenital
Strengths and limitations of the study

- First study of its kind exploring health-related quality of life (HRQOL) in surgical patients with congenital heart disease (CHD) through a mixed-methods approach in one of the low-income and middleincome countries.

- Qualitative assessment of the study will help identify unique, contextual sociodemographic HRQOL issues in patients with $\mathrm{CHD}$.

- Comparison of the HRQOL of patients with CHD with their age-matched siblings will control a number of potential confounders that could influence the study findings.

- Quality of life questionnaires will be used in the study setting for the first time; hence, there could be cross-cultural bias.

- Patient recruitment from a single centre might limit the generalisability of the study findings.

conditions such as congenital heart disease (CHD) occur, there is the potential for substantial impact across the lifespan, especially given that almost $90 \%$ of patients with CHD survive until adulthood. ${ }^{2}$ Though advancement in medical and surgical management has decreased mortality and increased survival, the majority of CHD surgical procedures are palliative, improving symptoms, but leaving residual issues such as arrhythmias, heart failure, protein enteropathy, potential for further operations and pacemaker insertion. $^{3}$

Health-related quality of life (HRQOL) is an important consideration for patients with CHD following surgery as routine clinical measurements may not always truly reflect the disease symptoms, treatment burden, functional limitations or the need for adjustment to disability that a person may experience. HRQOL is complex as it requires understanding of an individual's perception and experiences of their health-related issues 
and their perceived level of adaptation and coping. Additionally, it is strongly suggested that HRQOL be assessed by using both generic and disease-specific questionnaires. ${ }^{4}$

As a research tool, HRQOL assessments in CHD provide validated measures of functional data that correlate with findings from standardised clinical assessment tools. ${ }^{5}$ In addition, HRQOL provides a good benchmark for assessing the benefit of surgical and other therapeutic interventions and reveals where the best patient-sensitive gains can be made.

As a clinical tool, assessment of HRQOL provides important data on individual patients' current health status. Poor HRQOL findings reflect poor functional status that, in turn, impacts on patients' quality-adjusted life years (QALYs). Poor HRQOL also results in higher utilisation of healthcare services ${ }^{6}$ and may lead to later difficulties in securing and maintaining employment. ${ }^{7}$ For these reasons, HRQOL screening should be routine in CHD populations and is recommended by the Centers for Disease Control and Prevention ${ }^{8}$ and the American Heart Association. ${ }^{9}$

Uptake of routine HRQOL screening has been slow, and this may be due to clinicians' beliefs about availability and usability of screening tools, methodological concerns and pragmatic considerations, the latter being addressed by more recent electronic formats. ${ }^{10}$ A lack of HRQOL monitoring that reveals patient and parent perspectives to practitioners misses an opportunity for crucial collaborative development of a patient-centred care plan that may enhance adherence to treatment recommendations. ${ }^{11}$ HRQOL screening in diverse clinical settings has led to more frequent quality of life (QOL) discussions between patients and clinicians, including symptoms, function in activities of daily living and treatment satisfaction, ${ }^{10}$ and such discussions may provide the basis for increased psychological support. ${ }^{12}$

While survival of patients with CHD hasimproved, a number of studies from high-income countries have reported lower overall HRQOL in surgical patients with CHD compared with normal cohorts. ${ }^{1314}$ These are expressed in multiple domains including physical, ${ }^{13-16}$ motor, ${ }^{17}$ emotional, ${ }^{14-18}$ social, ${ }^{14151718}$ school, ${ }^{1415}$ cognitive and psychosocial functioning ${ }^{13-15}$ in both patients' and parents' reports. When critiqued using the Newcastle-Ottowa Scale for Cross-sectional Studies ${ }^{19}$ and Gill and Feinstein's 10 criteria for evaluating HRQOL research, ${ }^{20}$ CHD HRQOL reports are generally strong in their application of rigorous statistical methods, use of a control group and their representativeness for the target population. Limitations include conceptual definition of HRQOL used, providing opportunity for patients to highlight HRQOL domains important to them, and poor justification for the sample sizes used. ${ }^{14} 1621-23$ This body of work has also been criticised for lack of conceptual rigour and poor attention to reporting of inclusion criteria. ${ }^{24}$

There is some evidence that the presence of resources such as having educated parents, ${ }^{25}$ living with family and having higher socioeconomic status ${ }^{22}$ is associated with better HRQOL of patients with CHD. While these available resources are important contributors to QOL in CHD, a crucial consideration in HRQOL is the extent of the underlying disease and the impact of related symptoms. This is well illustrated in the Wilson and Cleary model of HRQOL (modified by Ferrans et al) ${ }^{26}$ (figure 1). Based on this theoretical model, this study will explore the relationship of biological function, symptoms, functional status and general health perception along with the individual and environmental characteristics or the available resources with HRQOL.

The influence of resource availability on HRQOL suggests that study findings on CHD surgical outcomes conducted in high-income countries (HIC) are likely to differ from those conducted in low-income and middle-income countries (LMIC). For example, in Pakistan, more than 50\% of the population fall below the poverty line and literacy rates are low at $60 \%$. This mismatch is a problem because CHD is common in LMIC where birth rates are high. ${ }^{27}$

The number of people with CHD can be difficult to characterise because the terminologies of incidence and

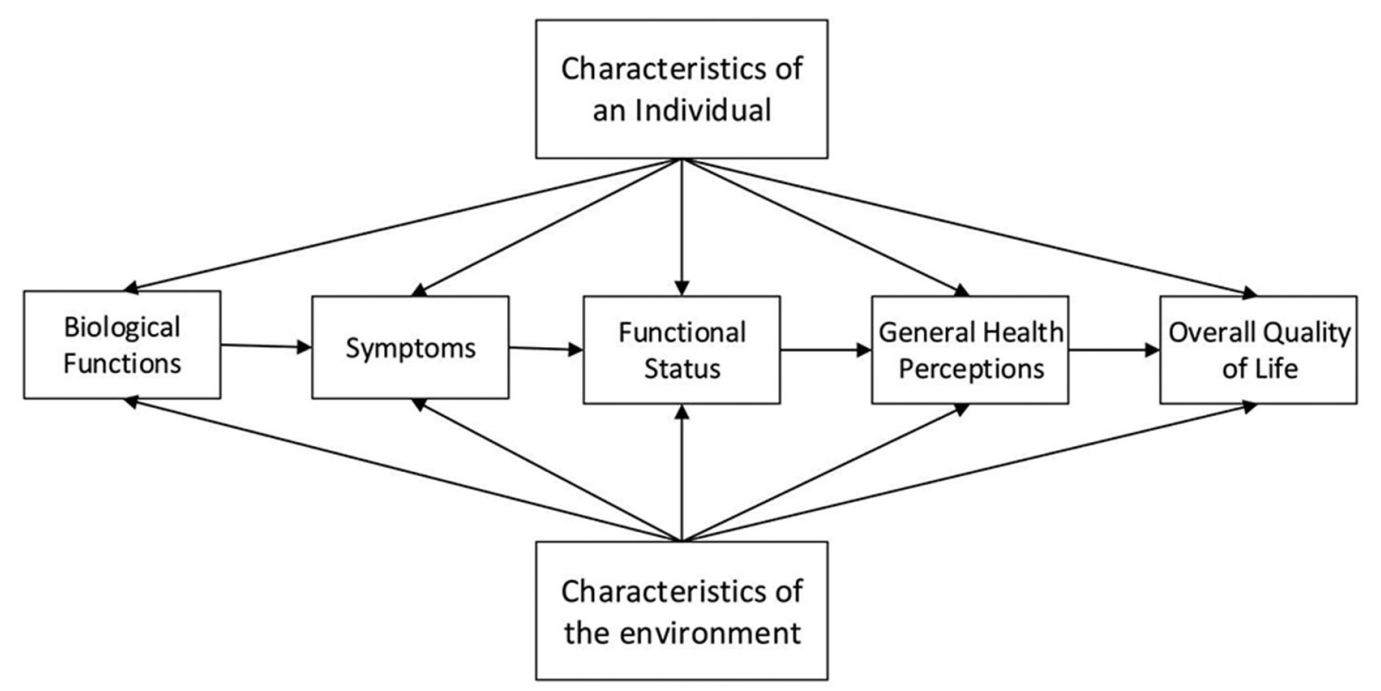

Figure 1 Revised Wilson and Cleary model for health-related quality of life. 
prevalence are often used interchangeably. ${ }^{27} \mathrm{CHD}$ varies across continents, with Asia having the highest CHD incidence of $9.3 / 1000$ live births. ${ }^{28}$ While incidence is often similar across continents, prevalence varies, depending on fertility and birth rates. Inferring Asian CHD incidence to Pakistan, it is estimated that 50000 children with CHD are born every year in the country. ${ }^{29}$ The true prevalence may be higher because of the incomplete data registry.

Pakistan has a lack of resources for health provision, where for a population of 180 million, there are only 5 hospitals providing paediatric cardiology services, 25 paediatric cardiologists and 8 paediatric cardiac surgeons. ${ }^{30}$ Adding to the difficulty in providing health services, $62 \%$ of the population lives in rural areas where accessibility, availability and affordability issues lead to late diagnosis, referral and surgery as well as difficulties with CHD follow-up. ${ }^{31-33}$ At times, families sacrifice their jobs and sell their assets to enable their child to have life-saving surgery for CHD. ${ }^{34}$ Therefore, it is essential to investigate HRQOL following CHD surgery in this context, to identify needs and arrange for optimal and targeted support within the available resources.

We were unable to find literature on HRQOL for patients with CHD from LMIC. This represents a major gap in our understanding for this population. Considering the differences in sociodemographics and the impact of constrained resources on late presentation and surgery, HRQOL may differ according to a country's economic status. The scarce literature available on CHD outcomes from LMIC does not explore QOL or longterm outcomes and, rather, focuses on 30-day mortality and initial surgical outcomes. ${ }^{33} 35-38$ This is problematic, given that CHD is a disease requiring life-long care, and surgery is palliative rather than curative. ${ }^{39}$ In routine clinical practice, patients identified at high risk of long-term, poor HRQOL provide a smaller, targeted population for more costly evaluation measures (eg, neurocognitive testing and cardiac imaging). HRQOL screening therefore has potential to inform more cost-effective care planning and, in turn, to increase QALYs.

Given the contextual differences between HIC and LMIC such as Pakistan, it is crucial that the HRQOL of this population is compared with an appropriate group. In this case, age-matched healthy siblings are ideal because of the shared sociodemographics controlling for a number of confounding factors. ${ }^{40}{ }^{41}$ Furthermore, an insight into parents' and children's experiences following CHD surgery will provide essential information to help develop appropriate processes for care that respond to the specific context.

This study intends to determine HRQOL for people following CHD surgery compared with age-matched healthy siblings. It also strives to identify predictors of HRQOL in surgical patients with CHD. Additionally, we will describe the everyday experiences of patients and families after CHD surgery to illuminate our quantitative findings. The study findings will provide an important benchmark against which future efforts may be compared, within individual countries and across countries internationally. We do expect to see some differences in HRQOL in the Pakistani setting compared with results from HIC. If this study can detect and explain these differences, then it is indeed adding value to the pre-existing literature and highlights the need of conducting work that is contextual. In addition, the qualitative arm of the study will help us identify contextual modifiers of HRQOL in patients with CHD in a country like Pakistan.

\section{METHODS}

Aim

This paper aims to describe the study methodology to explore the HRQOL in postoperative patients with CHD in Pakistan.

\section{Research questions}

The study aims to address the following research questions:

In a tertiary care hospital in Pakistan following CHD surgery:

1. What is the HRQOL in patients with CHD compared with their age-matched sibling?

2. What are the predictors (preoperative, intraoperative, postoperative, postdischarge and sociodemographics) of HRQOL in patients with CHD?

3. What are the experiences of patients with CHD and their parent, related to surgery, recovery and living with CHD?

\section{Study design}

The study uses the convergence model of a concurrent triangulation mixed-methods design, ${ }^{42}$ which has been recommended for outcomes research. ${ }^{43}$ This includes quantitative and qualitative approaches that complement each other and focus on data collection in parallel (figure 2). The simultaneous data collection was also chosen for pragmatic purposes, making the most of participant contact, given the long distances people travelled to take part.

The quantitative data will be compared by age-matched siblings to control confounding factors like genetics, sociodemographics and sociocultural factors. ${ }^{44}$ While the qualitative and quantitative data are analysed separately, the results are converged to compare and contrast findings during the final interpretation. The concurrent triangulation used in this study will provide an in-depth and comprehensive understanding of the complex phenomenon of HRQOL in postsurgery patients with CHD, and the added value of sibling comparison makes this study unique.

\section{Study setting and participants}

This study will be conducted at The Aga Khan University Hospital (AKUH), which is a tertiary care private hospital in Karachi, Pakistan. Approximately 1400 patients with CHD have received surgery since the site began 


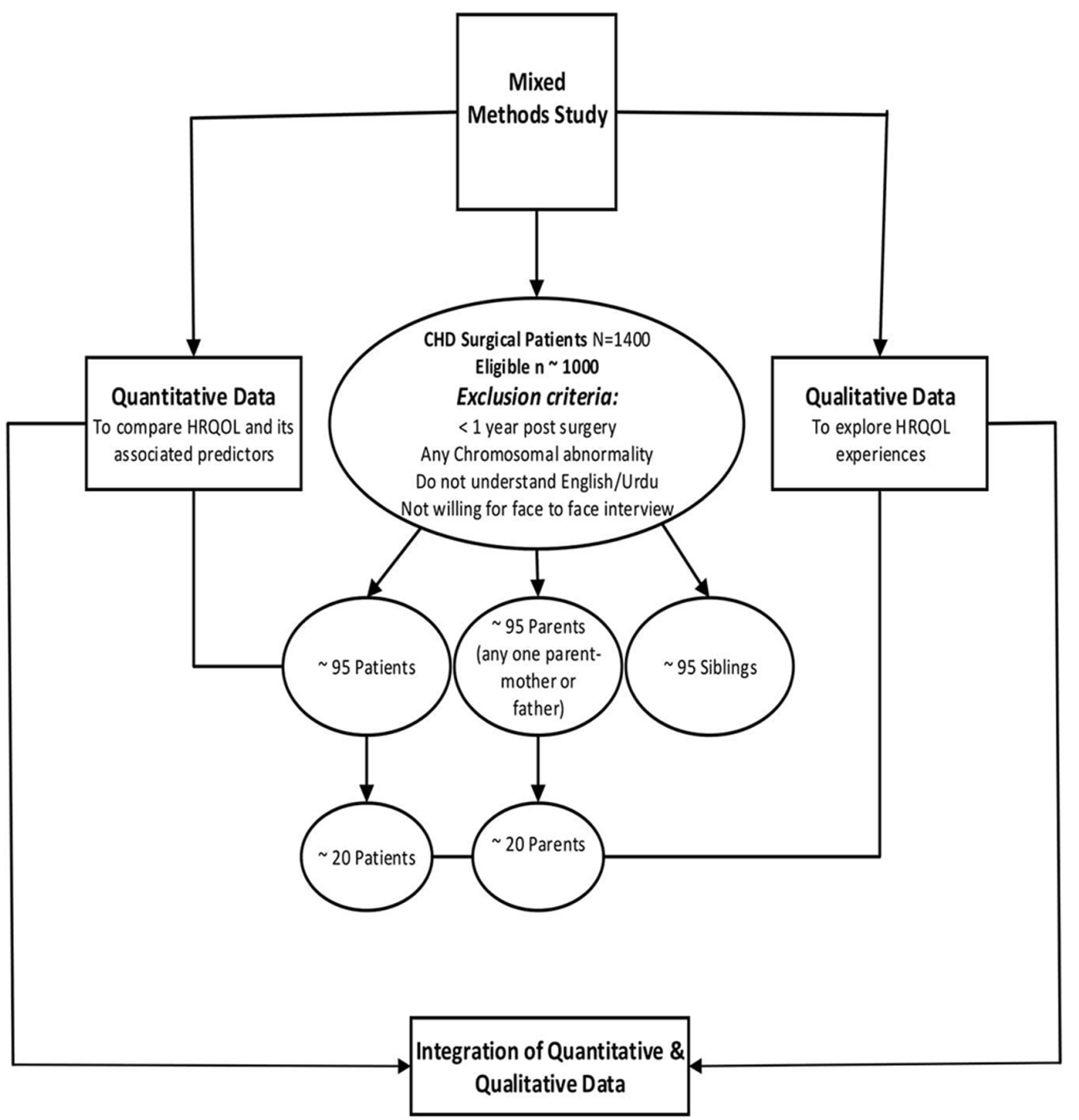

Figure 2 Study flow diagram. Health-related quality of life (HRQOL) in surgery patients with congenital heart disease in Pakistan: a mixed-methods study.

its structured paediatric cardiac surgery programme (2008-2015). Patients who are at least 1-year post-CHD surgery, with no chromosomal abnormality, are able to understand English and/or Urdu and are willing to attend a face-to-face interview will be eligible for this study. Patients, their age-matched siblings and parents will be recruited for the quantitative study, and a subsample of the enrolled patients and parents will be recruited for the qualitative interviews. One parent (either the father or mother) would be invited for both quantitative and qualitative data collection. The anticipated time for recruitment is 6 months, and we will continue sampling for this time period even if the target sample size is reached.

\section{Quantitative arm}

The quantitative arm uses a prospective cross-sectional descriptive study design to compare HRQOL of postoperative patients with CHD with their age-matched siblings. It will also identify the predictors of HRQOL.
Sample size and sampling

All surgical patients who meet the eligibility criteria (estimated $n=1000$ ) will be approached. Patient report, as the primary focus of this study, was used for sample size calculation. In the absence of sample size calculations in previously published HRQOL CHD studies, a small Cohen's d effect size ${ }^{45}$ of 0.2 between CHD and their age-matched sibling, a power of 0.8 and a probability of 0.05 was considered. Sample size was calculated on the basis of 11 predictors to be examined, which suggested a minimum sample size of 95 per group. ${ }^{46}$ While we hope to see effect sizes that suggest clinically meaningful changes, we have powered our study to detect small effect sizes. This is because, even when the magnitude of a change in QOL is small, it may be experienced as important by the patient. $^{47}$

A convenience sample of patients, their age-matched siblings and parents will be recruited from the study setting. Though the use of age-matched siblings can 
control a number of confounding factors that could potentially impact the study findings, it may be viewed by some as a limitation, with criticism of overmatching leading to sampling bias. ${ }^{44}$ In addition, there could be other impacts of CHD diagnosis and treatment on the entire family. One option is to recruit controls from a larger community, representative of the general population. There are, however, reasons for our age-matched sibling approach. Many of the patients being recruited will belong to periurban slums, and these communities are diverse ethnically, economically and socially. ${ }^{48}$ Families living in the same neighbourhood may still have different sociodemographic experiences. ${ }^{49}$ Thus, use of unrelated controls in a country like Pakistan may actually defeat the purpose of removing the sociodemographic confounders. Based on this, we choose to select sibling controls. Our choice is further directed by the financial and time constraints of recruiting an unrelated sample: based on our clinical experience, siblings frequently attend clinics with patients with CHD. A paired, matched analysis and conditional logistic regression will be used to control this potential threat to external validity. ${ }^{50}$

Eligible patients and their addresses will be identified through the hospital's patient database. The primary physician of the eligible patients will be informed about this study, and a study information letter will be sent to the eligible patients and their parents (if age less than 18 years) or to patients alone (18 years and above). The letter will invite potential participants to call the research team on the telephone numbers provided if they are interested in taking part in the study. A face-to-face interview time will then be scheduled. Additionally, potential patient and/or parent participants will be approached in the hospital's outpatient paediatric cardiology clinic and provided with information regarding the study and allowed time to consider this. An assent will be obtained from the patients ( $5-18$ years) in addition to an informed consent from parents and from patients ( $>18$ years). Potential participants will be assured that they are not obliged to participate. In addition, if they participate, they may withdraw their participation or their data at any time without giving a reason, and this will not affect their medical treatment or relationship with hospital staff.

\section{Data collection}

HRQOL data will be collected using questionnaires. Overall HRQOL will be assessed using the PedsQL 4.0 Generic Core Scale. This scale has 23 items and includes domains for physical (8 items), emotional (5 items), social (5 items) and school (5 items) functioning. The cognitive domain will be assessed by PedsQL Cognitive Functioning Scale, which has six items.

The PedsQL 3.0 Cardiac Module will also be used to identify HRQOL specific to the cardiac problem. It is a 27-item scale that focuses on the domains of heart problems (7 items), treatment ( 5 items), perceived physical appearance (3 items), treatment anxiety ( 4 items), cognitive problems ( 5 items) and communication (3 items).
Both the questionnaires have been widely used in patients with $\mathrm{CHD}^{15}$ 21-23 51-54 and other health problems ${ }^{55} 56$ and have well-established reliability. ${ }^{56} 57$ They are available for populations of different ages as well as their parents/proxy for young children (5-7 years), children ( $8-12$ years), teenagers (13-18 years), young adults (18-25 years) and adults ( $>26$ years). There is also a proxy/parent version only for toddlers ( $2-4$ years) given developmental limitations on self-report for children younger than 5 years of age. The questionnaires have very similar response items and scoring. Permission to use the questionnaires has been obtained from the Mapi Research Trust. Questionnaires have been translated to Urdu, which is the local language spoken in Pakistan, and back translated into English by a qualified translator to verify the translation credibility.

These questionnaires capture patient experiences for the last month. Both patients and parents will be asked to rate their HRQOL from 0 to 4 with 0 being 'never a problem' and 4 being 'almost always a problem'. Responses will be reverse scored and linearly transformed to $0-100$ with the higher the score indicating better HRQOL. If more than $50 \%$ of the items in the scale are missing, the scale scores will be considered as missing data. The Psychosocial Health Summary Score will be calculated by adding the sum of the items over the number of items answered in the Emotional, Social and School Functioning Scales. A mean score of the individual domains will be calculated by adding the sum of the items over the number of items answered. The total score will be the sum of all the items over the number of items answered on all scales.

Sociodemographic data will be collected from patients, parents and siblings to characterise the sample's current age, gender, socioeconomic background, educational status, occupation and family structure. In addition, patients will be asked for their current cardiac medication details (if any) and New York Heart Association functional class, which is a validated tool widely used both in research and clinical settings to assess the functional status of cardiac patients. ${ }^{23}$

Patients' clinical and surgical data will be retrieved from their medical files. It will include CHD diagnosis, age at diagnosis and surgery, surgical procedure, number of surgical procedures, cardiopulmonary bypass time and length of stay. In addition, the CHD surgical procedure will be assigned a Risk Adjustment for Congenital Heart Surgery (RACHS-1) score to identify the inpatient surgical mortality risk for the cardiac surgery performed. ${ }^{3758-60}$

\section{Data analysis}

Data will be described using means, standard deviation (SD) and frequency and numbers according to the level of the variable for the demographic, clinical and surgical indicators. Adjusted mean differences will be reported for HRQOL between the person with CHD and their matched siblings to control for the confounders. A p Value of 0.05 will be considered as significant. The cut-offs for PedsQL 
HRQOL scores reported in the literature are from HIC, which may not be applicable to LMIC settings. Therefore, the study would consider 1 SD below the population mean as a clinically meaningful cut-off for lower HRQOL as has been suggested by Varni $e t a l^{61}$ and used by Uzark et $a l^{52}$ Comparisons will also be made between patient and parents and between different CHD diagnoses. For the cardiac module, comparison will only be made between different CHD diagnoses.

Multiple regression analysis will be used to create models for total HRQOL, cardiac specific HRQOL and cognitive function. As identified in the literature, ${ }^{13} 18222562$ the variables used in the analyses will include gender, socioeconomic background, parental education, family structure, cardiac lesion, age at surgery, number of surgeries, cardiopulmonary bypass time, RACHS scale, length of stay and current cardiac medications. The model significance is defined as $\mathrm{p}<0.05$.

\section{Qualitative arm}

Qualitative data on health-related experiences will be collected on a subsample of surgical patients with CHD and their parents, drawn from the sample in the quantitative arm.

\section{Sample size and sampling}

Stratified purposive sampling ${ }^{63}$ by age group will guide recruitment of participants. A minimum of 5-7 patients from each age group (13-18 years, 19-25 years, 25+ years) and parents of patients from each age group (1-4 years, 5-12 years, $13-18$ years) will be invited to participate in the qualitative arm of the study. Final numbers will be guided by a process of data saturation where major themes are richly described and there are no new themes developing. We would further enhance credibility of the qualitative data by participation equity for patients by gender, age and type of surgery. An additional written informed consent will be obtained for all individuals participating in the interviews.

\section{Data collection}

Qualitative data will be collected using individual interviews based on a semistructured interview guide. This guide has been designed based on the literature and in discussion with experts in this field, that is, a paediatric cardiologist (BSH), two nurse academics with research expertise in Cardiology (RG) (JG) and a PhD Student (LAL) who is also an experienced specialist nurse in paediatric cardiology. Topics addressed during the interview include the patient/parent's perception of HRQOL related to CHD surgery, identification of the domains of HRQOL that are most important to them during recovery, the areas where the impact of the CHD surgery has been most and least beneficial, the problems/challenges faced and their perceived needs for the future (box). The semistructured interview guide is translated in Urdu and back translated in English by a second researcher to verify the translation credibility. The interviews will be conducted

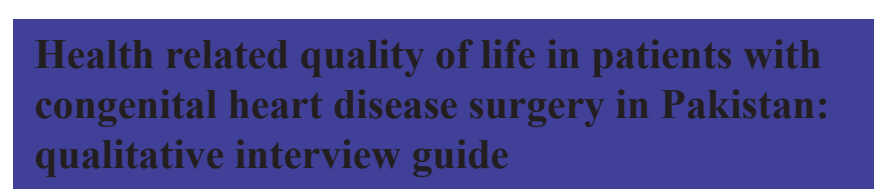

\section{For patients}

1. In your view, what is quality of life?

2. How do you see your disease, that is, congenital heart disease and surgery?

3. How does the community and society view your congenital heart disease and surgery?

4. What is the most disturbing or bothersome aspect of your surgery?

5. How do you deal with these issues and problems?

6. What are some of the concerns that are very important for you to discuss for your future years?

7. What do you think are the needs of patients with congenital heart disease with surgery and what would you like to suggest to the healthcare professionals to address those needs?

For parents

1. In your view, what is quality of life for your child?

2. How do you see your child's disease, that is, congenital heart disease and surgery?

3. How does the community and society view your child's congenital heart disease and surgery?

4. What is the most disturbing or bothersome aspect of your child's surgery?

5. How do you/your child deal with these issues and problems?

6. What are some of the concerns that are very important for you to discuss for your child's future years?

7. What do you think are the needs of patients with congenital heart disease with surgery and what would you like to suggest to the healthcare professionals to address those needs?

by a single researcher (LAL), trained in qualitative techniques, in a dedicated quiet room at the study setting. The interviews will be conducted in English or Urdu based on the participants' preference and will be audio-recorded for accuracy. Interviews will be transcribed verbatim in the language in which they were recorded. Interview transcripts transcribed in Urdu will then be translated into English for analysis.

\section{Data analysis plan}

Interview data will be analysed using directed content analysis $^{64}{ }^{65}$ guided by the Wilson-Cleary theoretical model of HRQOL ${ }^{26}$ (figure 1). In directed content analysis, the initial coding is guided by an existing theory or research findings. As researchers read and reread the data, their understandings are grouped under elements of the theoretical framework and are interpreted against the researchers' understandings of the social, cultural, clinical and demographic profile of the participants, to arrive at a description of the participants' experience of the phenomenon. Two members from the research team will review the transcripts and collaborate on analysis to enhance the credibility of findings. Rigour of the qualitative component will be enhanced by the application of Elo $e$ t $a$ 's 'Checklist to Improve the Trustworthiness of a 
Content Analysis Study' to the preparation, organisation and reporting phases. ${ }^{66}$

\section{Ethics and dissemination}

This study has received approval from the ethics review committee of the study setting. Informed consent will be obtained from patients and their parents both orally and in writing, and participants will be offered a choice to receive that information in English or Urdu. Assent will also be obtained from patients' of age $5-18$ years. Patients and/or their parents will be provided with transportation costs through the support of the paediatric department of AKUH.

While the risks associated with this study are negligible, it is possible that a participant may become distressed while telling their story. In this instance, we would follow the participant distress protocol developed for this study. Data confidentiality and archiving will be maintained as per Good Clinical Practice Guidelines. ${ }^{67}$ Study findings will be disseminated through peer-reviewed publications and presentation at national and international conferences.

\section{Anticipated challenges}

We anticipate certain challenges while conducting this study. Approaching the patients by phone or mail could be challenging due to changes in contact details that might not have been updated in the hospital database. In addition, recruitment directly from the hospital clinic may be difficult due to irregular clinic presentations. Transcribing the qualitative interviews into Urdu and then arranging their translation and back-translation provides an added layer of complexity. However, initial translation from Urdu to English will be conducted by one bilingual researcher (LAL) to avoid interpretation bias. The English version of key qualitative data will then be given to a second Urdu-speaking researcher and cardiology physician (BSH) to translate back to Urdu. The two Urdu texts will then be checked for congruence of meaning and any differences carefully considered and negotiated for the most accurate English equivalent. ${ }^{68}$ Using the descriptive analytical method of content analysis, we will keep the level of interpretation very 'close to the data ${ }^{69}$ further reducing the potential for misinterpretation.

Our strong clinical connections to the study setting, track of patients at the outpatient clinics and outreach trips to more remote referral areas will inform our interpretation of qualitative data and will assist in maximum patient recruitment.

Despite these study challenges, we look forward to the findings of this study that will provide much-needed understanding of the needs of patients and families who experience CHD and surgery within a LMIC. This will allow a more nuanced development of interventions and resources to meet these particular needs.

\section{CONCLUSION}

This study aims to determine the HRQOL of people who have surgery to address CHD and to identify associated HRQOL predictors in Pakistan. Considering the differences in sociodemographic variables and the surgical outcomes between HIC and LMIC, it is likely that HRQOL may differ between countries and that differences may be influenced by individual, social and cultural contexts. HRQOL of surgical patients with CHD will be assessed as self-report and will be compared with their age-matched healthy siblings and their parents' assessment. Triangulation of data will add richness and depth to our understanding of the factors and processes related to HRQOL following CHD surgery. This may provide a focus for CHD care planning and resource allocation in the LMIC of Pakistan.

Contributors LAL is a PhD candidate who conceived the study, contributed to the study design, drafted and revised the manuscript. BSH contributed to the study design and provided critical feedback on the manuscript drafts. JG contributed to the study design and provided critical feedback on the manuscript drafts. KA contributed to the study design, provided critical feedback on the manuscript drafts and assisted in formatting. AA contributed to the study design, provided critical feedback on the manuscript drafts and assisted in formatting. RG contributed to the study design and provided critical feedback on the manuscript drafts.

Funding This project is unfunded. However, as this study is a component of a PhD thesis, LAL received an International Postgraduate Research Scholarship and an Australian Postgraduate Award from The University of Sydney, Australia, to pursue Doctoral studies.

Competing interests None declared.

Patient consent Obtained.

Ethics approval This study has received ethics approval from The Aga Khan University, Pakistan, 3737-Ped-ERC-15.

Provenance and peer review Not commissioned; externally peer reviewed.

Open Access This is an Open Access article distributed in accordance with the Creative Commons Attribution Non Commercial (CC BY-NC 4.0) license, which permits others to distribute, remix, adapt, build upon this work non-commercially, and license their derivative works on different terms, provided the original work is properly cited and the use is non-commercial. See: http://creativecommons.org/ licenses/by-nc/4.0/

(c) Article author(s) (or their employer(s) unless otherwise stated in the text of the article) 2017. All rights reserved. No commercial use is permitted unless otherwise expressly granted.

\section{REFERENCES}

1. Claessens P, Moons P, de Casterlé BD, et al. What does it mean to live with a congenital heart disease? A qualitative study on the lived experiences of adult patients. Eur J Cardiovasc Nurs 2005;4:3-10.

2. Brickner ME, Hillis LD, Lange RA. Congenital heart disease in adults. N Engl J Med Overseas Ed 2000;342:256-63.

3. Engelfriet P, Boersma E, Oechslin E, et al. The spectrum of adult congenital heart disease in Europe: morbidity and mortality in a 5 year follow-up period. The Euro heart survey on adult congenital heart disease. Eur Heart J 2005;26:2325-33.

4. Wiebe S, Guyatt G, Weaver B, et al. Comparative responsiveness of generic and specific quality-of-life instruments. J Clin Epidemiol 2003;56:52-60.

5. Buratti S, Ryberg C, Broberg M, et al. Do self- and proxy reports of cognitive problems reflect intellectual functioning in children and adolescents with congenital heart defects? Front Pediatr 2016;4.

6. Cuthbertson BH, Roughton S, Jenkinson D, et al. Quality of life in the five years after intensive care: a cohort study. Crit Care 2010;14:R6.

7. Ladouceur M, Iserin L, Cohen S, et al. Key issues of daily life in adults with congenital heart disease. Arch Cardiovasc Dis 2013;106:404-12. 
8. Oster ME, Riehle-Colarusso T, Simeone RM, et al. Public health science agenda for congenital heart defects: report from a Centers for Disease Control and Prevention experts meeting. J Am Heart Assoc 2013;2:e000256.

9. Rumsfeld JS, Alexander KP, Goff DC, et al. Cardiovascular health: the importance of measuring patient-reported health status: a scientific statement from the American Heart Association. Circulation 2013;127:2233-49.

10. Giesinger J, Kemmler G, Meraner V, et al. Towards the implementation of quality of life monitoring in daily clinical routine: Methodological issues and clinical implication. Breast Care 2009;4:148-54.

11. Marino BS, Tomlinson RS, Wernovsky G, et al. Validation of the pediatric cardiac quality of life inventory. Pediatrics 2010;126:498-508.

12. Lithoxopoulou H, Zarogoulidis K, Bostantzopoulou S, et al. Monitoring changes in quality of life in patients with lung cancer by using specialised questionnaires: implications for clinical practice. Support Care Cancer 2014;22:2177-83.

13. Knowles RL, Day T, Wade A, et al. Patient-reported quality of life outcomes for children with serious congenital heart defects. Arch Dis Child 2014;99:413-9.

14. Mellion K, Uzark K, Cassedy A, et al. Health-related quality of life outcomes in children and adolescents with congenital heart disease. J Pediatr 2014:164:781-8.

15. Idorn L, Jensen AS, Juul K, et al. Quality of life and cognitive function in Fontan patients, a population-based study. Int J Cardiol 2013;168:3230-5.

16. Neal AE, Stopp C, Wypij D, et al. Predictors of health-related quality of life in adolescents with tetralogy of Fallot. $J$ Pediatr 2015;166:132-8.

17. Spijkerboer AW, Utens EM, De Koning WB, et al. Health-related Quality of Life in children and adolescents after invasive treatment for congenital heart disease. Qual Life Res 2006;15:663-73.

18. Landolt MA, Valsangiacomo Buechel ER, Latal B. Health-related quality of life in children and adolescents after open-heart surgery. J Pediatr 2008;152:349-55.

19. Higgins JPT, Green S. eds. Cochrane Handbook for Systematic Reviews of Interventions, Version 5.1.0: The Cochrane Collaboration, 2011. (updated Mar 2011).

20. Gill TM, Feinstein AR. A critical appraisal of the quality of quality-oflife measurements. JAMA 1994;272:619-26.

21. Eagleson KJ, Justo RN, Ware RS, et al. Health-related quality of life and congenital heart disease in Australia. $J$ Paediatr Child Health 2013;49:856-64.

22. Garcia Guerra G, Robertson CM, Alton GY, et al. Quality of life 4 years after complex heart surgery in infancy. $J$ Thorac Cardiovasc Surg 2013;145:482-8.

23. Kwon EN, Mussatto K, Simpson PM, et al. Children and adolescents with repaired tetralogy of fallot report quality of life similar to healthy peers. Congenit Heart Dis 2011;6:18-27.

24. Drakouli M, Petsios K, Giannakopoulou M, et al. Determinants of quality of life in children and adolescents with CHD: a systematic review. Cardiol Young 2015;25:1027-36.

25. Larsen SH, McCrindle BW, Jacobsen EB, et al. Functional health status in children following surgery for congenital heart disease: a population-based cohort study. Cardiol Young 2010;20:631-40.

26. Ferrans CE, Zerwic JJ, Wilbur JE, et al. Conceptual model of healthrelated quality of life. J Nurs Scholarsh 2005;37:336-42.

27. Hoffman Jle. The global burden of congenital heart disease. Cardiovasc J Afr 2013;24:141-5.

28. van der Linde D, Konings EE, Slager MA, et al. Birth prevalence of congenital heart disease worldwide: a systematic review and metaanalysis. J Am Coll Cardiol 2011;58:2241-7.

29. Humayun KN, Atiq M. Clinical profile and outcome of cyanotic congenital heart disease in neonates. J Coll Physicians Surg Pak 2008;18:290.

30. Society PPC. Pakistan Pediatric Cardiology Society: For Children born with CHD, 2015.

31. Mocumbi AO. The challenges of cardiac surgery for African children. Cardiovasc J Afr 2012;23:165-7.

32. Mocumbi AO, Lameira E, Yaksh A, et al. Challenges on the management of congenital heart disease in developing countries. Int $J$ Cardiol 2011;148:285-8.

33. Rashid U, Qureshi AU, Hyder SN, et al. Pattern of congenital heart disease in a developing country tertiary care center: Factors associated with delayed diagnosis. Ann Pediatr Cardiol 2016;9:210.

34. Mughal AR, Sadiq M, Hyder SN, et al. Socioeconomic status and impact of treatment on families of children with congenital heart disease. J Coll Physicians Surg Pak 2011;21:398-402.
35. Mirabel M, Lachaud M, Offredo L, et al. Cardiac surgery in lowincome settings: 10 years of experience from two countries. Arch Cardiovasc Dis 2017;110:82-90

36. Reddy NS, Kappanayil M, Balachandran R, et al. Preoperative determinants of outcomes of infant heart surgery in a limitedresource setting. Semin Thorac Cardiovasc Surg 2015;27:331-8.

37. Bakshi KD, Vaidyanathan B, Sundaram KR, et al. Determinants of early outcome after neonatal cardiac surgery in a developing country. $J$ Thorac Cardiovasc Surg 2007;134:765-71.

38. Sadiq M, Nazir M, Sheikh SA. Infective endocarditis in childrenincidence, pattern, diagnosis and management in a developing country. Int J Cardiol 2001;78:175-82.

39. Webb G, Mulder BJ, Aboulhosn J, et al. The care of adults with congenital heart disease across the globe: current assessment and future perspective: a position statement from the International Society for Adult Congenital Heart Disease (ISACHD). Int J Cardiol 2015;195:326-33.

40. Manlhiot C, Knezevich S, Radojewski E, et al. Functional health status of adolescents after the Fontan procedure - comparison with their siblings. Can J Cardiol 2009;25:S294-300.

41. Knowles R, Veldtman G, Hickey EJ, et al. Functional health status of adults with tetralogy of Fallot: matched comparison with healthy siblings. Ann Thorac Surg 2012;94:124-32.

42. Creswell JW, Plano Clark VL, Gutmann ML, et al. Advanced mixed methods research designs. In: Tashakkori A, Teddlie C, eds. Handbook of mixed methods in social and behavioral research. Thousand Oaks, CA: Sage Publisher, 2003:209-40.

43. Curry LA, Nembhard IM, Bradley EH. Qualitative and mixed methods provide unique contributions to outcomes research. Circulation 2009;119:1442-52.

44. Frisell T, Öberg S, Kuja-Halkola R, et al. Sibling comparison designs: bias from non-shared confounders and measurement error. Epidemiology 2012;23:713-20.

45. Lakens D. Calculating and reporting effect sizes to facilitate cumulative science: a practical primer for t-tests and ANOVAs. Front Psychol 2013;4:863.

46. Soper D. Statistics Calculator. Accessed: 01 April, 2017 https://www. danielsoper.com/statcalc/calculator.aspx?id=1.

47. Middel B, van Sonderen E. Statistical significant change versus relevant or important change in (quasi) experimental design: some conceptual and methodological problems in estimating magnitude of intervention-related change in health services research. Int J Integr Care 2002;2.

48. Muller N, Werner P, Kelcey JG. Urban biodiversity and design, 2010:497-517.

49. Van Ham M, Manley D, Bailey N. Understanding neighbourhood dynamics: New insights for neighbourhood effects research. Springer, 2012:163.

50. Pearce N. Analysis of matched case-control studies. BMJ 2016;352:i969.

51. Tahirović $\mathrm{E}$, Begić $\mathrm{H}$, Nurkić $\mathrm{M}$, et al. Does the severity of congenital heart defects affect disease-specific health-related quality of life in children in Bosnia and Herzegovina? Eur J Pediatr 2010;169:349-53.

52. Uzark K, Jones K, Slusher J, et al. Quality of life in children with heart disease as perceived by children and parents. Pediatrics 2008:121:e1060-7.

53. Mellion K, Uzark K, Cassedy A, et al. Quality of life scores in children and adolescents with congenital heart disease are lower than in the healthy pediatric population. Congenit Heart Dis 2010;5:517.

54. Sand P, Kljajić M, Sunnegårdh J. The reliability of the Pediatric Quality of Life Inventory 3.0 Cardiac Module for Swedish children with congenital heart defects. Nord Psychol 2013;65:210-23.

55. Limbers CA, Heffer RW, Varni JW. Health-related quality of life and cognitive functioning from the perspective of parents of school-aged children with Asperger's syndrome utilizing the PedsQL. J Autism Dev Disord 2009;39:1529-41.

56. Varni JW, Limbers CA, Sorensen LG, et al. PedsQL Cognitive functioning scale in pediatric liver transplant recipients: feasibility, reliability, and validity. Qual Life Res 2011;20:913-21.

57. Varni JW, Seid M, Kurtin PS. PedsQL 4.0: reliability and validity of the Pediatric quality of life inventory version 4.0 generic core scales in healthy and patient populations. Med Care 2001;39:800-12.

58. Nina RV, Gama ME, Santos AM, et al. Is the RACHS-1 (risk adjustment in congenital heart surgery) a useful tool in our scenario? Rev Bras Cir Cardiovasc 2007;22:425-31.

59. Larsen SH, Pedersen J, Jacobsen J, et al. The RACHS-1 risk categories reflect mortality and length of stay in a Danish population of children operated for congenital heart disease. Eur J Cardiothorac Surg 2005;28:877-81. 
60. Jenkins KJ. Risk adjustment for congenital heart surgery: the RACHS-1 method. Semin Thorac Cardiovasc Surg Pediatr Card Surg Annu 2004;7:180-4.

61. Varni JW, Burwinkle TM, Seid M, et al. The PedsQL 4.0 as a pediatric population health measure: feasibility, reliability, and validity. Ambul Pediatr 2003;3:329-41.

62. McCrindle BW, Williams RV, Mitchell PD, et al. Relationship of patient and medical characteristics to health status in children and adolescents after the Fontan procedure. Circulation 2006;113:1123-9.

63. Patton MQ. Qualitative evaluation and research methods. Beverly Hills, CA: Sage Publications, 1990:169-89.

64. Hsieh HF, Shannon SE. Three approaches to qualitative content analysis. Qual Health Res 2005;15:1277-88.
65. Graneheim UH, Lundman B. Qualitative content analysis in nursing research: concepts, procedures and measures to achieve trustworthiness. Nurse Educ Today 2004;24:105-12.

66. Elo S, Kääriäinen M, Kanste $\mathrm{O}$, et al. Qualitative content analysis: a focus on trustworthiness. Sage Open 2014;4:1-10.

67. Emanuel EJ, Wendler D, Grady C. What makes clinical research ethical? JAMA 2000;283:2701-11.

68. Ballantyne PJ, Yang M, Boon H. Interpretation in cross-language research: tongues-tied in the health care interview? J Cross Cult Gerontol 2013;28:391-405.

69. Elo $\mathrm{S}$, Kyngäs $\mathrm{H}$. The qualitative content analysis process. J Adv Nurs 2008;62:107-15. 